\title{
PREDICTION OF THE AGEING OF RUBBER USING THE CHEMI- LUMINESCENCE APPROACH AND ISOCONVERSIONAL KINETICS
}

\author{
F. Käser ${ }^{1,2,3 *}$ and B. Roduit \\ ${ }^{1}$ ACL Instruments AG, Industriestr. 11, 3210 Kerzers, Switzerland \\ ${ }^{2}$ Berne University of the Arts, Fellerstr. 11, 3027 Berne, Switzerland \\ ${ }^{3}$ AKTS AG, TechnoArk 3, 3960 Siders, Switzerland
}

\begin{abstract}
A common scepticism towards the application of many product formulations results from the fact that their long-term stability is difficult to predict. In the present study we report on a new approach of kinetic analysis of the oxidation reactions of natural rubbers with and without stabiliser in an oxygen atmosphere at moderate temperatures using CL measurements carried out on a newly-developed instrumentation. The kinetic parameters of the oxidation process, calculated from the chemiluminescence's signals by means of the differential isoconversional method of Friedman, were subsequently applied for the simulation of the rubber aging under different temperature profiles. The presented results are the first stage of research by using the chemiluminescence method to measure the oxidative aging of rubber and predicting the life time of rubber items.
\end{abstract}

Keywords: chemiluminescence, isoconversional kinetics, lifetime prediction, oxidation

\section{Introduction}

It is known that most of the organic materials readily react with the oxygen even at ambient temperature; therefore the oxidative degradation is a severe material-engineering problem. Monitoring, or better, predicting the stability of organic and especially polymeric materials against oxidation is therefore of paramount importance. Commonly applied analytical methods such as measuring oxidation induction time (OIT) or oxidation onset temperature (OOT) using differential scanning calorimetry (DSC) or other conventional thermoanalytical methods are unsuitable for long-term prediction of oxidative behaviour because of the use of elevated temperatures during these experiments: the high temperatures may invoke reaction pathways which are different from those encountered under the conditions of use.

Oxidation processes, leading to the cleavage of the polymer chains, occur randomly and stealthily. They may not be detected for years undergoing the rapid, autocatalytic acceleration once the reaction has reached a critical stage. Once the reduction of the molecular mass has reached a critical threshold both adhesion and cohesion properties are critically affected what, in turn, leads to the loss of required bond properties.

The oxidation of materials in the solid-state generally starts on the surface and the oxidation progress is mainly diffusion controlled. The general overview of commonly applied examination methods and mechanism of the oxidation is given by Feller [1], Zweifel [2] and Scheirs [3]. The accelerated ageing is normally initialised by extreme environmental conditions. During the induction period, stabilisers are consumed while the organic matter remains stable, maintaining its original properties. At the end of the induction period, when the concentration of stabilisers reaches a sub-critical level, oxidative decay starts and the substance's properties change. Frequently, oxidation reactions are self-accelerating (auto-oxidation) and the reaction progress may rapidly increase after the induction period.

The most common methods to test the kinetics of thermooxidation of organic substances are thermal analysis methods like differential thermal analysis (DTA), DSC or thermogravimetry (TG). Substances are tested using isothermal or non-isothermal temperature profiles under an oxidative atmosphere, and the OIT or OOT are determined when the heat flow (DSC, DTA) starts to be exothermal or the sample mass loss starts (TG). In some special applications, these procedures are standardised (e.g. for automotive oils, cable insulations or polyolefines).

Especially for industrial and commercial applications, OIT and OOT determination procedures are widely applied. Their advantages include an easy sample preparation, short measurement periods and established methods of data evaluation. A significant disadvantage of these short-time experiments lays in 
the application of the high experimental temperatures, generally above $180^{\circ} \mathrm{C}$. The reasons of the use of such temperatures are following: (i) firstly to make sure that the oxidation starts within around $2 \mathrm{~h}$ and (ii) to provide a distinct signal being larger than the baseline noise: the sensitivity of conventional TA instruments can be too low to record the beginning of the oxidation reaction. The evaluation of the bad correlation of OIT and OOT data with the observed long-term stabilities under normal environmental conditions is reported in [4-6]. Depending on the properties of the substance, one or several phase transitions can occur between the temperature ranges at which the oxidative characteristic has been measured and those one for which the oxidative properties has been predicted (life-time determination). It seems to be obvious that the degradation kinetics may change for different low- and high-temperature phases and the extrapolation of high temperature experimental results to ambient temperature can be of little value.

In such situation the alternative methods, based on the experiments carried out at low temperatures, should be applied for the characterisation of the long-term stability of organic substances. One of very suitable methods is the chemiluminescence $(\mathrm{CL})$.

\section{Chemiluminescence}

Luminescence is a term used for various phenomena, originating from electronically excited states. Luminescence is a 'cold light', not an incandescent light. The emission of photons results from the relaxation of excited electrons (triplet-state) into their groundstate. This may be a quite quick process: the delay between the excitation and light emission is at least $10^{-10} \mathrm{~s}$. Chemiluminescence (CL) includes all luminescence phenomena resulting from chemical reactions [7]. The fact that organic substances undergoing oxidation emit light has been recognized already at the second half of XIX century [8]. In the past few years, chemiluminescence has gained wide acceptance as a sensitive method to study the oxidative degradation of organic solid substances [9-11].

\section{Principles of chemiluminescence in organics}

The emission of light during the oxidative degradation process of the organics is a part of the reaction course. The first step is the formation of unstable alkyl radicals, which immediately scavenge the oxygen from the atmosphere to form peroxy radicals. These react further and transform into different species in an accelerating degradation cycle (auto-oxidation, left part of Fig. 1). It is normally attributed to a transition of excited triplet-carbonyl-functions

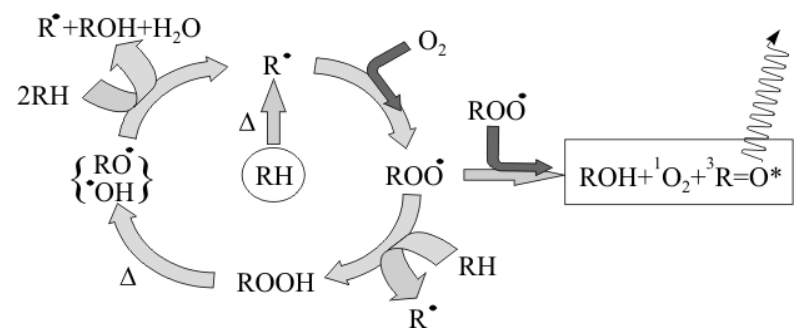

Fig. 1 Simplified scheme of auto-oxidation of organics including a Russel mechanism (from Lacey et al. [12])

$\left({ }^{3} \mathrm{R}=\mathrm{O}^{*}\right)$ into their ground state. The spectral range of the light emitted varies according to the type of substances involved. In most cases the chemiluminescence is observed in the short wave region of the visible spectrum from 380 to $450 \mathrm{~nm}$. However, there are well-known exceptions: the relaxation of ${ }^{1} \mathrm{O}_{2}$ can be detected in the infrared region at ca. $1200 \mathrm{~nm}$.

The required energy $\left(290-340 \mathrm{~kJ} \mathrm{~mol}^{-1}\right)$ may be supplied by basically three different chemical mechanisms:

- The combination of two peroxy radicals with concomitant fragmentation in a Russel mechanism [13] is strongly exothermal $\left(460 \mathrm{~kJ} \mathrm{~mol}^{-1}\right)$ [14]. The CL-emitter is an excited 'triplet' carbonyl function (right-hand part of Fig. 1).

- The direct homolysis of hydroperoxides followed by a cage reaction leads to an excited carbonylfunction and is combined with the evolution of $315 \mathrm{~kJ} \mathrm{~mol}^{-1}[15]$.

- The metathesis of alkoxy or peroxy radicals provides 374 and $323 \mathrm{~kJ} \mathrm{~mol}^{-1}$, respectively [16]. It has been shown, that the CL signal intensity reveals the existence of two kinetic stages during oxidative degradation of organic materials: The first one is correlated with the concentration of peroxide groups [17], the second stage corresponds to the oxidation propagation by the hydrogen abstraction responsible for the carbonyl formation [18].

\section{Advantages of chemiluminescence in monitoring oxidation of organics}

Compared to DSC and other conventional thermo-analytical methods, CL offers many advantages: due to its much higher sensitivity, experiments can be performed at much lower temperatures i.e. closer to the application related conditions. This fact is of the importance for the characterisation of substances with low temperature melting points, glass transitions, etc. The outstanding baseline stability of CL is of great benefit when performing long-term experiments [19]; moreover the CL-signal is related exclusively to the oxidation processes and therefore is not superposed by the signals resulting from the other reactions, in- 
cluding phase transitions. The instrumentation setup may be designed individually for special fields of applications and goals of research. The experiments can be performed with the sample masses as low as approx. $0.1 \mathrm{mg}$. A basic instrumentation would not be more expensive than a commercial DSC apparatus.

\section{Determination of the kinetic parameters - isoconversional analysis}

The noticeable weakness of the 'single curve' methods (determination of the kinetic parameters from single run recorded with one heating rate or isothermal condition only) has led to the introduction of 'multicurve' methods over the past few years, as discussed in the International ICTAC kinetics project [20-23].

Degradation reactions are often too complex to be described in terms of a single pair of Arrhenius parameters and the commonly applied set of reaction models. As a general rule, these reactions demonstrate profoundly multi-step characteristics. They can involve several processes with different activation energies and mechanisms. In such situation the reaction rate can be described only by complex equations, where the activation energy term is no more constant but is dependent on the reaction progress $\alpha$ ( $E \neq$ const. but $E=E(\alpha))$.

The isoconversional methods were introduced by Friedman [24] and Ozawa-Flynn-Wall [25, 26]. A detailed analysis of the various isoconversional methods (i.e. the isoconversional differential and integral methods) for the determination of the activation energy has been presented by Budrugeac [27]. The convergence of the activation energy values obtained by means of a differential method (Friedman) with those resulted from using integral methods (OzawaFlynn-Wall) comes from the fundamentals of the differential and integral calculus.

The differential isoconversional method of Friedman is based on the Arrhenius Eq. (1):

$$
\frac{\mathrm{d} \alpha}{\mathrm{d} t}=A \exp \left(-\frac{E}{R T(t)}\right) f(\alpha)
$$

where $f(\alpha)$ - the model function, $A$ - the pre-exponential factor, $E-$ the activation energy, $T$ - the temperature, $t$ - the time.

Friedman has applied the logarithm of the conversion rate $\mathrm{d} \alpha / \mathrm{d} t$ as a function of the reciprocal temperature at any conversion $\alpha$, Eq. (2):

$$
\begin{gathered}
\frac{\mathrm{d} \alpha}{\mathrm{d} t}=A(\alpha) \exp \left(-\frac{E(\alpha)}{R T(t)}\right) f(\alpha) \\
\ln \left(\frac{\mathrm{d} \alpha}{\mathrm{d} t}\right)=\ln (A(\alpha))-\frac{E(\alpha)}{R T(t)}+\ln (f(\alpha))
\end{gathered}
$$

As $f(\alpha)$ is a constant in the last term at any fixed $\alpha$, the logarithm of the conversion rate $\mathrm{d} \alpha / \mathrm{d} t$ over $1 / T$ shows a straight-line dependence with the slope of $m=-E / R$.

By the extension of the expression

$$
\ln \left(\frac{\mathrm{d} \alpha}{\mathrm{d} t}\right)=\ln \left(A^{\prime}(\alpha)\right)-\frac{E(\alpha)}{R T(t)}
$$

with

$$
A^{\prime}(\alpha)=A(\alpha) f(\alpha)
$$

one can predict the reaction rate or reaction progress having determined $A^{\prime}(\alpha)$ and $E(\alpha)$ using the following expression:

$$
\frac{\mathrm{d} \alpha}{\mathrm{d} t}=A^{\prime}(\alpha) \exp \left(-\frac{E(\alpha)}{R T(t)}\right)
$$

at any temperature profile such as isothermal, non-isothermal, stepwise, modulated temperature or periodic temperature variations, etc.

\section{Experimental}

The CL emission rate during oxidation of organic substances at ambient temperatures is too low to be detected. However, only moderate temperatures are required for providing detectable signals. The requirements for the oven applied are similar to those used in conventional TA measurements such as DSC or TG: the necessary is the exact control of the required temperature profile even in long-term experiments and a gas exchange facility. Additionally the sample compartment must be absolutely light-tight.

The instrumentations provided by AKTS-Chemiluminescence are fully automated and consist of cooled PMT with photon counting mode and an oven chamber in combination with an optical path including a shutter system (to protect the highly sensitive detection unit against extensive light during sample handling and to provide background measurements). The single-cell instrument is designed especially for sensitive measurements at moderate temperature conditions (isothermal and non-isothermal mode) additionally allowing carrying out the experiments under controlled relative humidity in the temperatures up to $95^{\circ} \mathrm{C}$. In addition, the CL instrument may be extended by a spectrometer to obtain the spectrometric information of the CL emission.

\section{Results and discussion}

In the present study we report on a new approach of kinetic analysis of the oxidation of organic solids at 
moderate temperatures using CL measurements carried out on newly-developed instrumentation. The kinetic characteristics of the oxidation process calculated from the chemiluminescence signals are subsequently applied for the prediction of the reaction progress under different temperature profiles.

The presented results depict the comparison of the oxidation reactions of natural rubbers with and without stabiliser in an oxygen atmosphere: This system is representative for many hot-melt formulations, especially HMPSA. The results shown in Fig. 2 illustrate the influence of the stabiliser (5\% Irganox $\left.{ }^{\mathrm{TM}} 565\right)$ on the oxidation behaviour of the rubber (cis-1,4-polyisoprene) during non-isothermal heating in the range $30-120^{\circ} \mathrm{C}$ with a rate of $0.0132 \mathrm{~K} \mathrm{~min}^{-1}$ in the oxygen atmosphere.

The set of experimental data under isothermal conditions in the range $80-120^{\circ} \mathrm{C}$ (Fig. 3) can be used for the evaluation of an Arrhenius relationship (Fig. 3, inset).

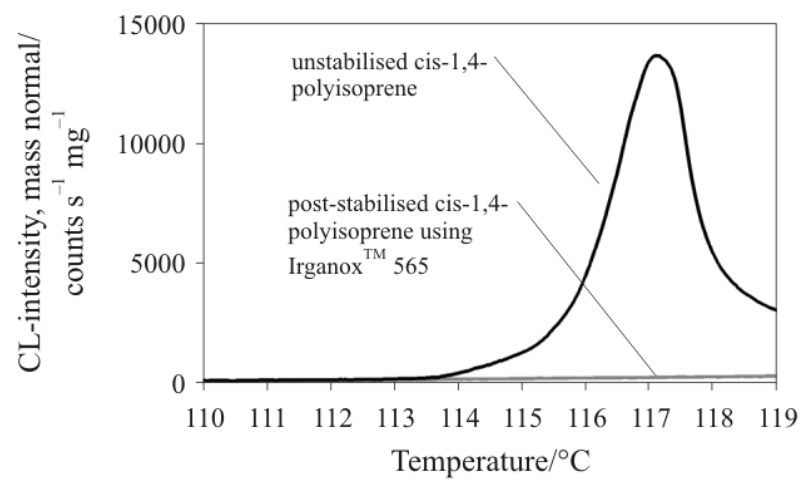

Fig. 2 CL signals recorded for the unstabilised and stabilised cis-1,4-polyisoprene during identical non-isothermal temperature profile in the oxygen atmosphere

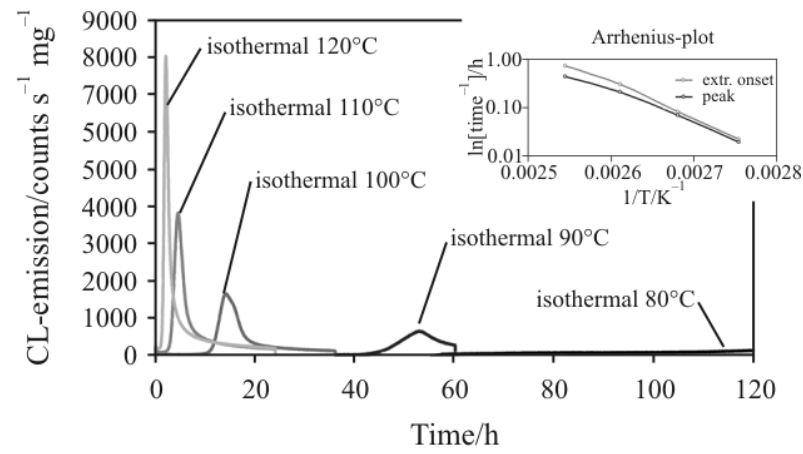

Fig. 3 Normalised CL-emission signals of unstabilised natural rubber (cis-1,4-polyisoprene) recorded during isothermal oxidation at $120,110,100,90,80^{\circ} \mathrm{C}$. The inset presents the Arrhenius relationship for the extrapolated onset and peak temperatures

\section{Application of an advanced kinetic analysis of $C L$-signals for the lifetime prediction}

Generally kinetic analysis of decomposition processes or any gas-solid reaction can be applied to any type of thermoanalytical data (DSC, DTA, TG, etc.) for the study of raw materials and products within the scope of research, development and quality assurance. The experimental procedure starts with the collection of the data directly related to the degradation kinetics such as mass loss or heat flow signals. These data are then used to determine the kinetic parameters such as activation energy $(E)$ or pre-exponential factor $(A)$ which allow predicting the reaction progress under various temperature modes. Generally, direct investigation of the reaction is very difficult at low temperatures (requiring very long measurement times), as well as under complex temperature profiles. Applying thermokinetics software (www.akts.com), the rate and the progress of the reactions can be predicted on the basis of various well-defined temperature profiles [28].

The CL signals collected during the oxidation of unstabilized and stabilised natural rubber under non-isothermal conditions at different heating rates were used for the determination of the kinetic parameters used later for the prediction of the reaction progress. The normalized reaction rates determined by AKTS-Thermokinetics software after correction and optimization of the baseline are depicted in Fig. 4.

The results of the evaluation of the kinetic parameters by the isoconversional method of Friedman are presented in Fig. 5.

Once the kinetic parameters are determined, they can be applied to predict the course of the oxidation under different temperature profiles. The presented results clearly indicate the oxidative induction period after which the rate of the oxidation accelerates rapidly. The prediction of the oxidation of the natural rubber under isothermal conditions at low temperatures $\left(4-28^{\circ} \mathrm{C}\right)$ is shown in Fig. 6.

The important goal of the investigation of the kinetics of the thermal decompositions is the need to determine the thermal stability of substances, i.e. the temperature range over which a substance does not decompose with an appreciable rate. The correct prediction of the reaction progress of materials which are unstable under ambient conditions requires accurate application in the calculations of both:

- the kinetic parameters

- the exact experimental temperature profile

The example showing the prediction of the properties of the rubber under more complicated temperature profile is depicted in Fig. 7 presenting the oxidation progress of natural rubber at $20^{\circ} \mathrm{C}$ when the tem- 

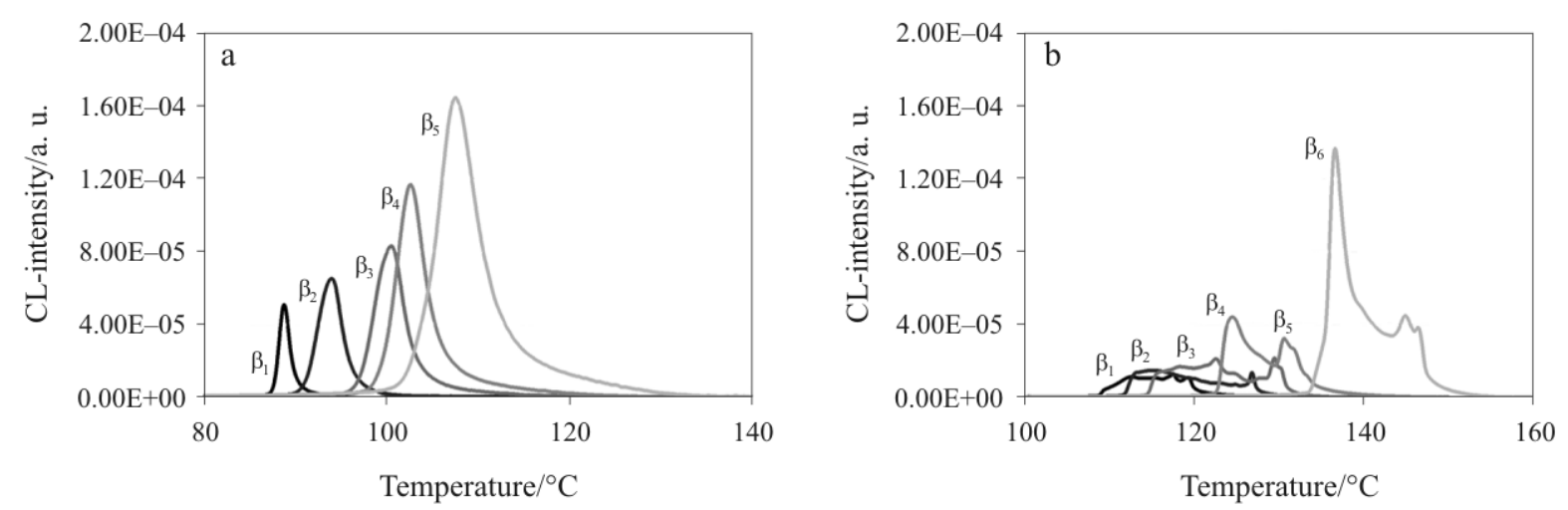

Fig. 4 Normalised CL signals recorded during oxidation of $a-$ unstabilized and $b$ - stabilized natural rubber with different heat ing rates $\beta\left(0.0054-0.073 \mathrm{~K} \mathrm{~min}^{-1}\right)$
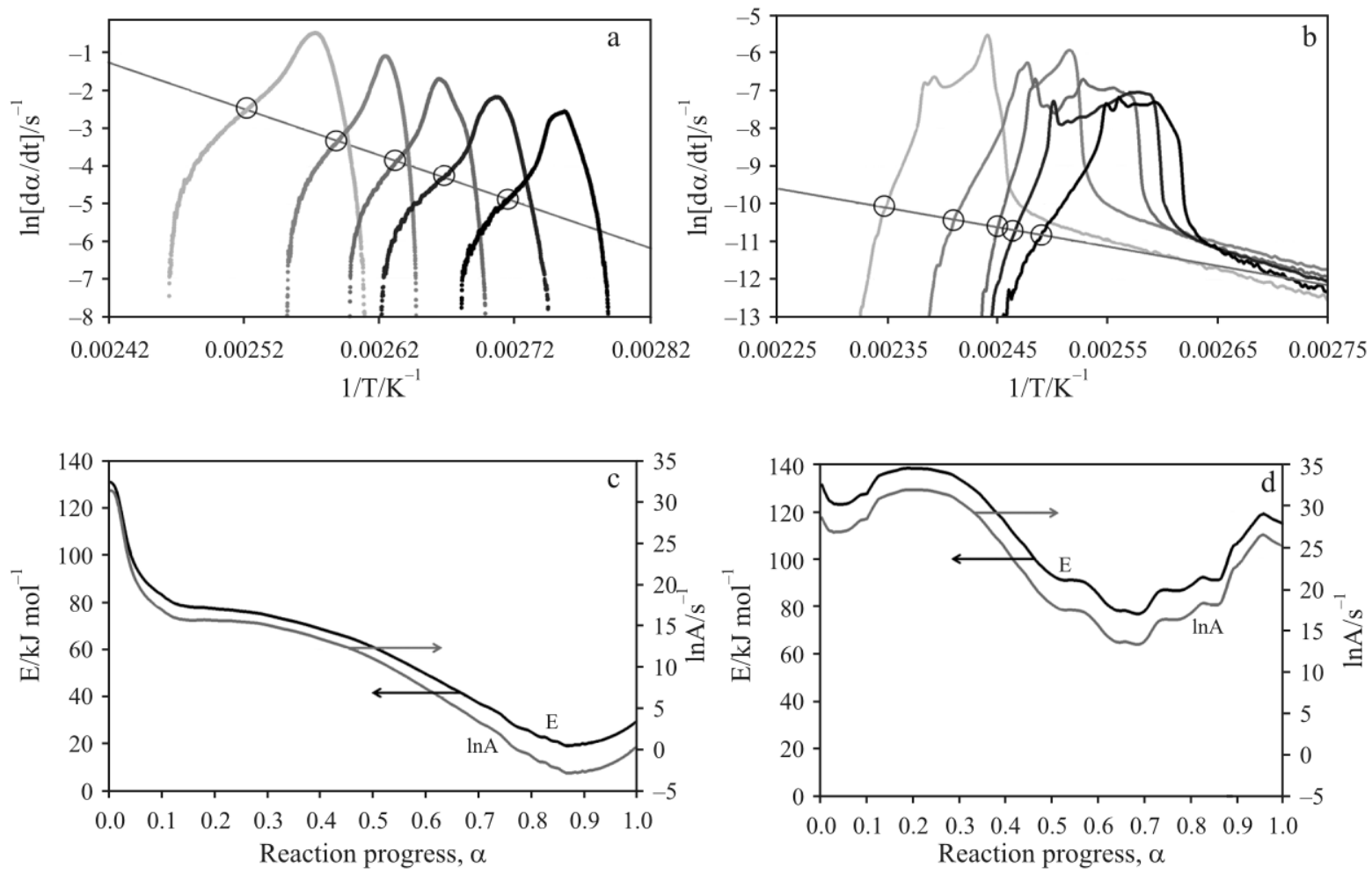

Fig. 5 a , b - Friedman differential isoconversional analysis of the long term oxidation process of $a-$ unstabilized and b-stabi lized natural rubber; $\mathrm{c}, \mathrm{d}$ - Dependence of the activation energy and the pre-exponential factor of natural rubber oxidation on the reaction progress $\alpha$ calculated by Friedman's differential isoconversional method: $\mathrm{c}-$ unstabilized and $\mathrm{d}-$ stabilized natural rubber

perature changes with the modulations of 0 and 1 to $7 \mathrm{~K}$ each $24 \mathrm{~h}$. The dependences shown in Fig. 7 indicate that even small temperature fluctuations can significantly change the stability of the substance: e.g. the amplitude of $10 \mathrm{~K}$ at $20^{\circ} \mathrm{C}$ lowers the oxidation stability of natural rubber by half of lifetime.

More generally, calculations can be achieved for any fluctuation of the temperature which makes possible the predictions of thermal stability properties for varying climates.
Exact consideration in the calculations of daily minimal and maximal temperature variations of worldwide climates provides therefore very valuable insight when interpreting and quantifying the reaction progress of materials subjected to atmospheric conditions. 

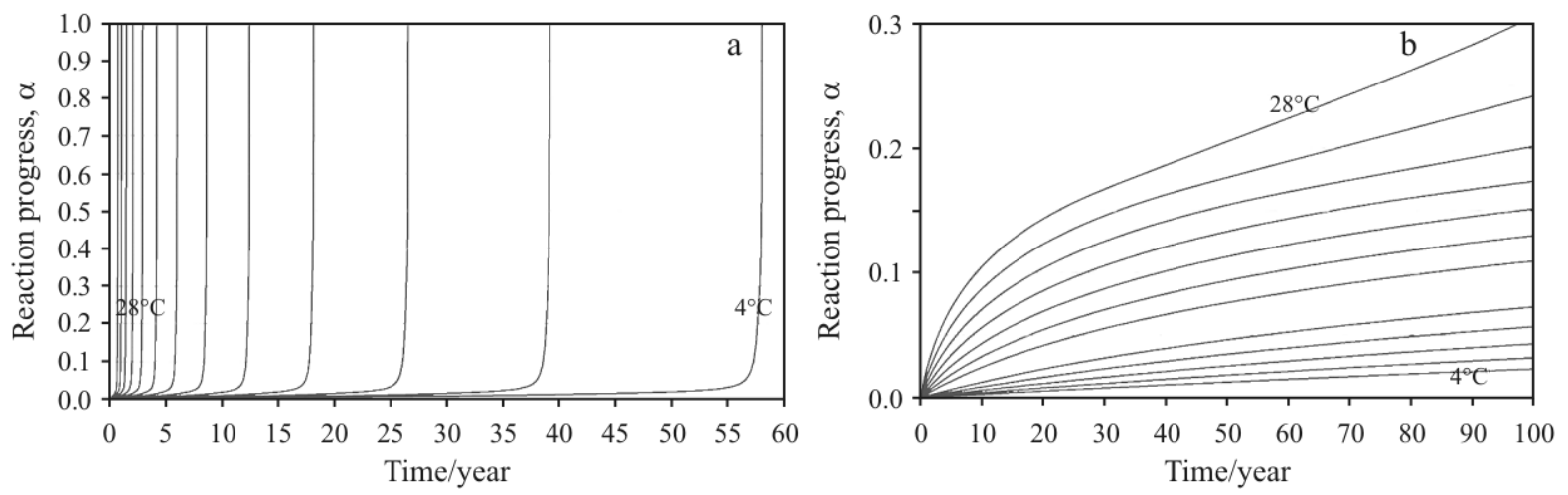

Fig. 6 Prediction of the oxidation progress of $\mathrm{a}$ - unstabilized and $\mathrm{b}$ - stabilized natural rubber at isothermal temperatures be tween 4 and $28^{\circ} \mathrm{C}$
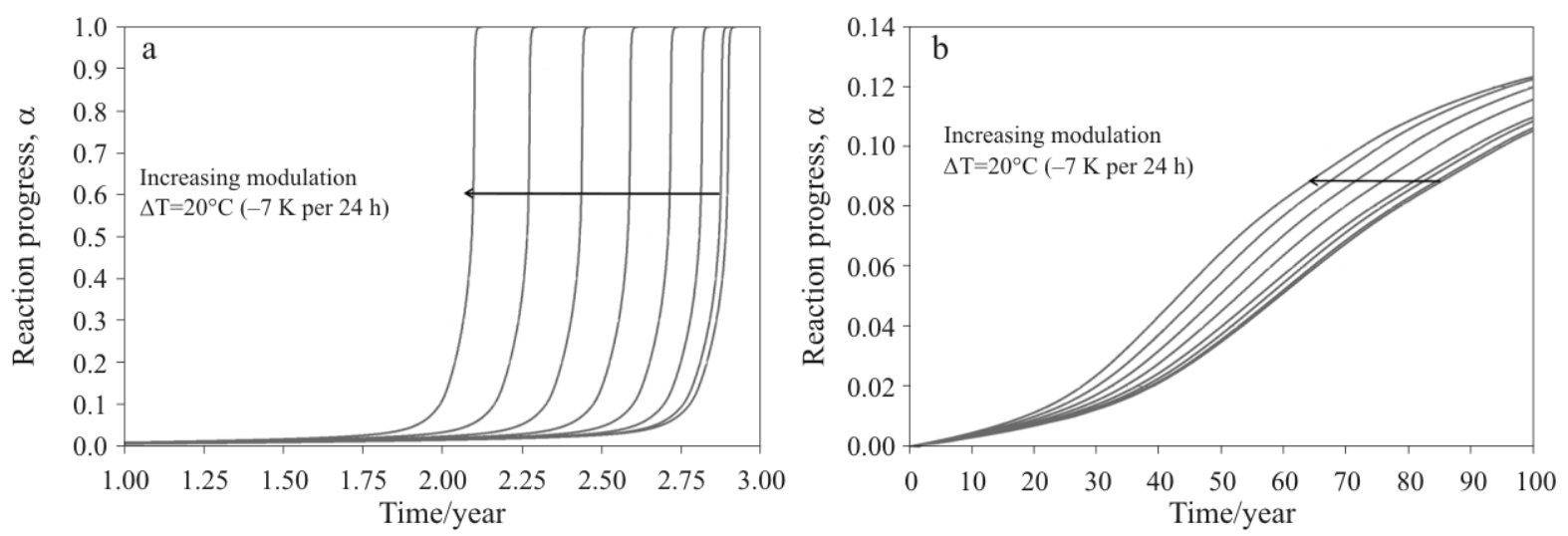

Fig. 7 Prediction of the oxidation progress of $a-$ unstabilized and $b-$ stabilized natural rubber at modulated isothermal tempera ture of $20^{\circ} \mathrm{C}$, amplitudes 0 and 1 to $7 \mathrm{~K}$ each $24 \mathrm{~h}$. Note that the average temperature is the same for all temperature pro files. Depending on the type of reactions and stabilization the effect of the temperature fluctuations can have significant influence on the reaction rate
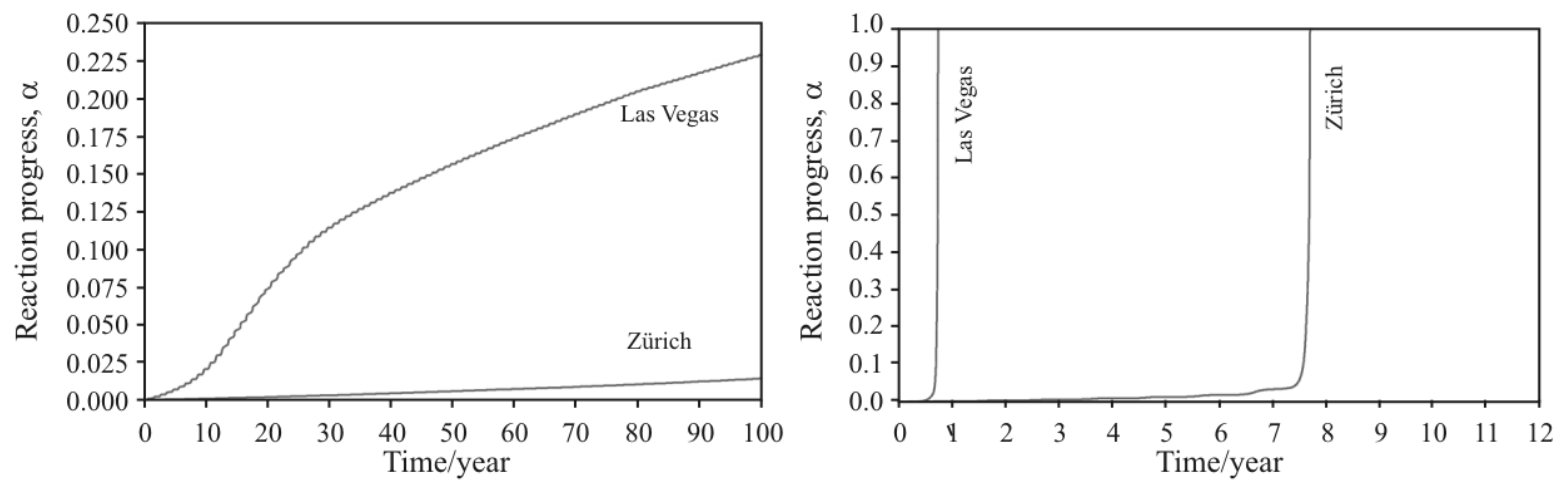

Fig. 8 Prediction of the oxidation progress of $a$ - unstabilized and b - stabilized natural rubber at different climatic locations (Las Vegas and Zürich)

\section{Conclusions}

Oxidative degradation of polymers can be monitored by the chemiluminescence method. This method is orders of magnitude more sensitive as compared to the conventional methods of thermal analysis such as
DSC, DTA or TG. The data acquired during the chemiluminescence experiments carried out iso- or nonisothermally can be evaluated by differential isoconversional kinetic analysis to obtain meaningful and accurate predictions of the life time of organic materials in temperature domains which are represen- 
tative for the life-cycle of the planned products. Within the same context the efficiency of stabilizers or influence of environmental factors such as relative humidity, UV-radiation, pollutants can be forecasted.

The presented results are the first stage of research by using the chemiluminescence method to measure the oxidative aging of rubber and predicting the life time of rubber items. The equipment used for the data collection was provided by AKTS-Chemiluminescence. It is a powerful tool, fully automated and supported by user-friendly yet powerful software. It enables $R \& D$ and production units to evaluate their products with respect to oxidative aging and to make meaningful predictions of the aging behaviour.

\section{References}

1 R. Feller, Accelerated Ageing, The Getty Conservation Institute, Marina del Rey 1994.

2 H. Zweifel., Plastic Additives Handbook, Verlag Carl Hanser, München 2001.

3 J. Scheirs, Compositional and Failure Analysis of Polymers, John Wiley \& Sons, Chichester 2000.

4 J. Pauquet, R. Todesco and W. Drake, $42^{\text {nd }}$ Int. Wire \& Cable Symp, St. Louis 1993, p. 77.

5 E. Kramer and J. Koppelmann, Polym. Eng. Sci., 27 (1987) 945.

6 F. Gugumus, Developments in Polymer Stabilisation - 8, G. Scott, Ed., Elsevier, London 1987, p. 239.

7 O.-A. Neumüller, Römpps Chemie-Lexikon, $8^{\text {th }}$ Ed., Franckh'sche Verlagshandlung, Stuttgart 1979-1988.

8 B. Radziszewski, Ber. D. Chem. Ges., 10 (1877) 70.

9 L. Zlatkevich, J. Polym. Sci. B, Polym. Phys., 28 (1990) 425.
10 M. Celina and G. George, Polym. Degrad. Stab., 40 (1993) 323.

11 L. Matisová-Rychlá and J. Rychly, J. Polym. Sci., 42 (2004) 648.

12 D. Lacey and V. Dudler, Polym. Degrad. Stab., 51 (1996) 101.

13 G. Russel, J. Am. Chem. Soc., 79 (1957) 3871.

14 R. Vasiliev, Prog. React. Kinet., 4 (1967) 305.

15 L. Reich and S. Stivala, Makromol. Chem., 103 (1967) 74.

16 E. Quinga and G. Mendenhall, J. Am. Chem. Soc., 105 (1983) 6520.

17 N. Billingham and E. Then, Polym. Degrad. Stab., 34 (1991) 263.

18 L. Audouin V. Bellenger, A. Tcharkhtchi and J. Verdu, Polymer Durability, R. Clough, N. Billingham and K. Gillen, Eds, American Chemical Society, Washington DC 1996, pp. 223-234.

19 J. Pospíšil, Z. Horák, J. Pilař, N. Billingham, H. Zweifel and S. Nešpůrek, Polym. Degrad. Stab., 82 (2003) 156.

20 M. E. Brown, M. Maciejewski, S. Vyazovkin, R. Nomen, J. Sempere, A. Burnham, J. Opfermann, R. Strey, H. L. Anderson, A. Kemmler, R. Keuleers, J. Janssens, H. O. Desseyn, C.-R. Li, T. B. Tang, B. Roduit, J. Malek and T. Mitsuhashi, Thermochim. Acta, 355 (2000) 125.

21 M. Maciejewski, Thermochim. Acta, 355 (2000) 145.

22 A. K. Burnham, Thermochim. Acta, 355 (2000) 165.

23 B. Roduit, Thermochim. Acta, 355 (2000) 171.

24 H. L. Friedman, J. Polym. Sci., C, (1965) 185.

25 T. Ozawa, Bull. Chem. Soc. Jpn., 38 (1965) 1881.

26 J. H. Flynn and L. A. Wall, Polym. Lett., 4 (1966) 323.

27 P. Budrugeac, J. Therm. Anal. Cal., 68 (2002) 131.

28 Advanced Kinetics and Technology Solutions Inc. Siders, Switzerland, http://www.akts.com

DOI: $10.1007 / \mathrm{s} 10973-007-8985-8$ 